EPJ Web of Conferences 88, 00006 (2015)

DOI: 10.1051/epjconf/ 20158800006

(C) Owned by the authors, published by EDP Sciences - SIF, 2015

\title{
In-medium effects around the Fermi energy
}

\author{
O. Lopez, D. Durand and G. Lehaut \\ (INDRA COLLABORATION)
}

\begin{abstract}
Laboratoire de Physique Corpusculaire, ENSICAEN, Université de Caen Basse Normandie, CNRS/IN2P3, F-14050 Caen, France
\end{abstract}

\begin{abstract}
We study nuclear stopping in central collisions for heavy-ion induced reactions in the Fermi energy domain (15-100 A MeV). Using the large dataset provided by the $4 \pi$ array INDRA, we determine that stopping can be directly related to the transport properties in the nuclear medium. By looking specifically at protons, we present a comprehensive body of experimental results concerning the mean free path, the nucleon-nucleon cross-section and in-medium effects in nuclear matter.
\end{abstract}

\section{Introduction}

Transport properties in nuclear matter contribute to the determination of the equation of state via the underlying in-medium properties of the nuclear interaction [1]- [8] as well as in the description of the supernova core collapse and the subsequent formation of a neutron star [9]. The knowledge of the dissipation mechanism for nuclear matter in HIC is related to the properties of the mean-field itself via the 1-body dissipation (nuclear friction and viscosity) and nucleon-nucleon $(N N)$ collisions via 2-body dissipation in the nuclear medium [10]. At low incident energy i.e. lower than the Fermi energy, where mean-field effects prevail, $N N$ collisions are strongly suppressed due to the fermionic nature of nucleons (Pauli blocking). At high 
incident energy, the mean-field becomes less and less attractive while $N N$ collisions become important [11-13]. Numerous theoretical approaches show that the cross section has to be properly renormalized in order to account for the effective $N N$ collision rate in $H I C[11,12,14]$ as depicted in theoretical works $[15-17]$. These latter show that the nucleon mean free path is large for $E_{\text {inc }} / A \leq 100 \mathrm{MeV}$, and decreases toward a saturation value $\lambda_{N N}=4-5 \mathrm{fm}$ around $E_{\text {inc }} / A \approx 100 \mathrm{MeV}$ [17]. In-medium effects and especially quenching factors for the $N N$ cross section are indeed largely unknown in the range $E_{\text {inc }} / A=10-100 \mathrm{MeV}$ [18] and have to be constrained experimentally.

\section{Experimental considerations}

\subsection{INDRA dataset}

In this analysis, we use the full INDRA dataset for symmetric or nearlysymmetric systems recorded at GANIL and GSI facilities. The experimental data are exclusive and corresponds to a nearly complete detection of all charged products of the reaction thanks to the powerful INDRA $4 \pi$ array [20]. The data cover a broad domain of incident energy, here from 15 up to $100 \mathrm{~A} \mathrm{MeV}$, and concern 42 systems with a total mass between 72 and 476 mass units. Isospin is here comprised between $N=Z$ and $N / Z \approx 1.6$. This constitutes, to our knowledge, the largest body of experimental data in the Fermi energy domain covered with the same setup.

\subsection{Event and particle selections}

In the following, we want to probe the degree of stopping in central collisions. We have then chosen to study the very dissipative collisions, that produce the highest charged particle multiplicities $M_{c h}$ as already done in [10]. The correlation between the isotropy ratio $R_{E}$ and $M_{c h}$ presents a saturation at the highest multiplicity values and then allow to define a multiplicity cut [10]. In order to probe the nucleon properties in nuclear medium, we have moreover to focus specifically on free nucleons. They indeed carry genuine information about $N N$ collisions, i.e. out of any coalescence phase nor clusterization into fragments occuring during the course of the collision [21]. 


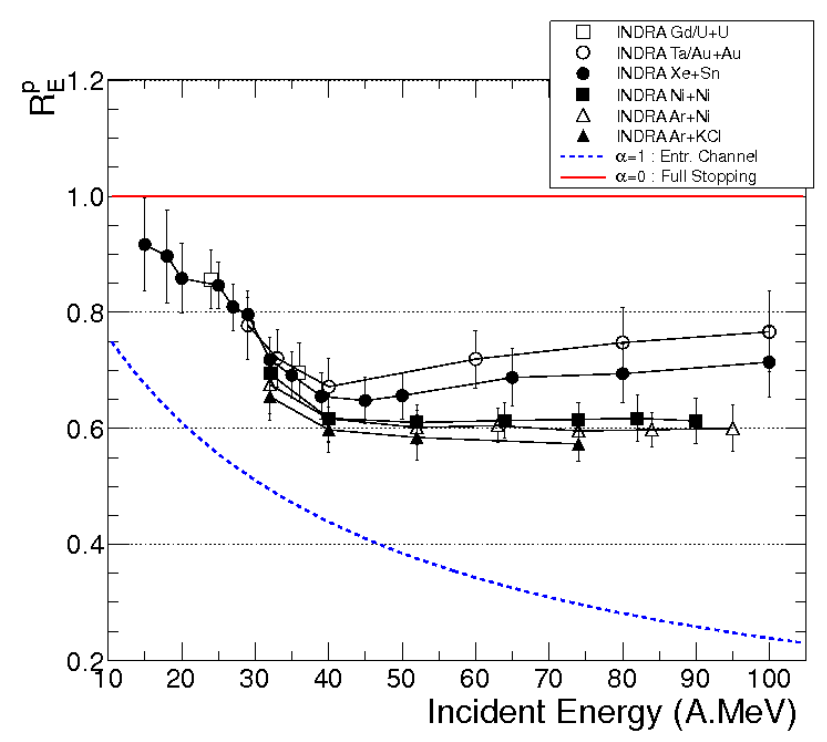

Figure 1: Mean isotropy ratio for protons $R_{E}^{p}$ as a function of energy. The symbols represent the different studied systems. The lower dashed curve represents the expected value for the entrance channel (no stopping) and the upper straight line for the full stopping (color online).

\section{Stopping ratio and $N N$ collisions}

Applying the protocol presented in the previous section, we compute the isotropy ratio $R_{E}^{p}$ for the 42 symmetric systems recorded with INDRA. The results are presented in Fig. 1 as a function of the incident energy.

The error bars in Fig. 1 correspond to the statistical errors supplemented by an estimate of the systematic errors coming from the experimental determination for $R_{E}^{p}$ [10]. To get more quantitative values for the stopping, the isotropy ratio is compared to two extreme values. They are computed by assuming two Fermi spheres in $p$-space separated by the relative momentum corresponding to $E_{\text {inc }}$, which is the incident energy and $\alpha$ a parameter equal to 1 for complete transparency (no dissipation, lower dashed curve in blue) and 0 for full stopping (upper straight line in red). A straightforward calculation for the isotropy ratio $R_{E}(\alpha)$ can be obtained analytically [10] and an estimate for the stopping reached in our dataset of central events is then given by the normalized quantity $\mathcal{S}$, called hereafter stopping ratio, and such as: $\mathcal{S}=\frac{R_{E}^{p}-R_{E}(\alpha=1)}{R_{E}(\alpha=0)-R_{E}(\alpha=1)}$. Using a simple Monte Carlo simulation describing in a semi-classical way $N N$ collisions, we find that $\mathcal{S}$ can be 
related to the ratio of accepted $N N$ collisions $\mathcal{C}$ by the following empirical formula: $\mathcal{C} \approx \mathcal{S}^{\beta\left(E_{i n c}\right)}$ with $\beta=1.32$ at $E_{i n c}=30 A \mathrm{MeV}$, and $\beta=0.74$ at $E_{\text {inc }}=110 A \mathrm{MeV}[23]$.

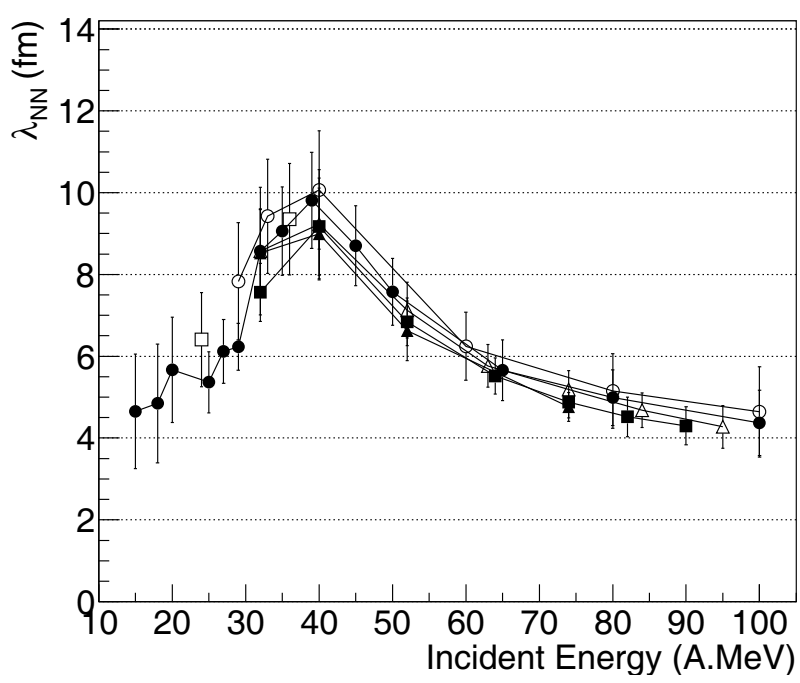

Figure 2: Mean free path for a nucleon in nuclear matter as a function of incident energy. Symbols are the same as for Fig. 1.

\section{In-medium effects}

To understand the mass hierarchy observed in Fig. 1, we scale the latter quantity $\mathcal{C}$ by $A_{\text {tot }}^{\gamma}, A_{\text {tot }}$ being the total mass number of the system, and $\gamma$ varying between $1 / 4-2 / 3$. The results show that for $\gamma \approx \frac{1}{3}$, all experimental points collapse on a single curve for the whole range of incident energy and for all systems; the agreement is somehow particularly impressive for incident energies above the Fermi energy [23]. This result suggests to define a characteristic quantity $A_{\text {tot }}^{1 / 3}$, homogeneous to a length, connected to the radial extent of the system formed in central collisions. From this, we can infer that the corresponding reduced value $\mathcal{C} / A_{\text {tot }}^{1 / 3}$ is related to the associated mean free path for $N N$ collisions. We postulate that the mean free path $\lambda_{N N}$ can be simply expressed as the inverse of $\mathcal{C}: \lambda_{N N} \approx L / \mathcal{C}$ where $L$ is a characteristic length proportional to $A_{\text {tot }}^{1 / 3}[23] . L$ can be interpreted as a quantity related to the average distance travelled by a nucleon. Applying 


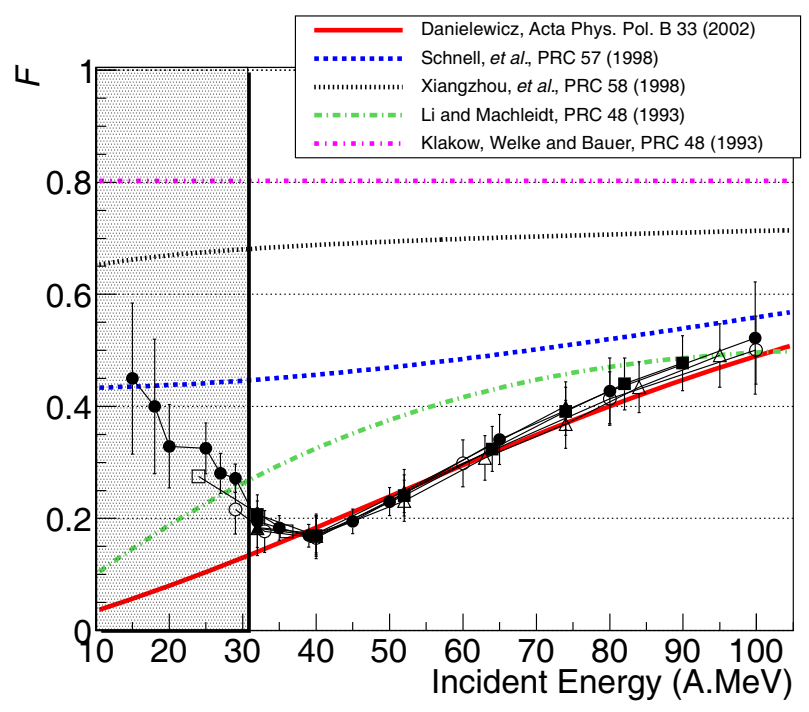

Figure 3: In-medium factor $\mathcal{F}=\sigma_{N N}^{\text {in-medium }} / \sigma_{N N}^{\text {free }}$ for the nucleon-nucleon cross section in nuclear matter. The different curves correspond to some parametrizations used in transport models. Symbols are the same as for Fig. 1.

this, we get the results for $\lambda_{N N}$ in Fig. 2 where we see that $\lambda_{N N}$ is maximum around $E_{\text {inc }}=35-40 \mathrm{~A} \mathrm{MeV}$, and reaches $\lambda_{N N}=9.5 \pm 2 \mathrm{fm}$.

This depicts the fact that the Pauli principle suppresses to a large extent $N N$ collisions at low incident energy and consequently increases the mean free path around the Fermi energy [11]. The decrease observed at lower incident energy is here attributed to mean-field effects, for which the dissipation mechanism is mainly provided by 1-body rather than 2-body dissipation , i.e. $N N$ collision. If we focus on the high energy domain, i.e. above the Fermi energy, we note a continuous decrease of $\lambda_{N N}$, whatever the system size, toward an asymptotic value corresponding to $\lambda_{N N}=4.5 \pm 1 \mathrm{fm}$ above $100 \mathrm{~A} \mathrm{MeV}$. These values are compatible with both experimental data $[24,25]$ and recent theoretical studies [17] around and above $100 \mathrm{~A} \mathrm{MeV}$. This agreement also suggests that the characteristic length $L$ is indeed closely related to the nuclear radius of the colliding system and justifies a posteriori our assumption.

From our estimated mean free paths, we can now determine the inmedium nucleon-nucleon cross section. In the following, we will compare the extracted in-medium cross-sections to the free values in vacuum. To disentangle the different in-medium effects, we evaluate the Pauli blocking 
by using the simple prescription of Kikuchi and Kawai [26]. We thus obtain nucleon-nucleon cross sections, out of Pauli effects, which have to be compared to the standard free values [27]. To get more quantitative results, we compute the in-medium factor $\mathcal{F}=\sigma_{N N}^{i n-\text { medium }} / \sigma_{N N}^{\text {free }}$. It is displayed in Fig. 3 where we restrict our discussion to the incident energy range $30-100$ $\mathrm{A} \mathrm{MeV}$.

The reduction factor $\mathcal{F}$ strongly evolves with incident energy, between 0.2 and 0.5 for the incident energy range $35-100 \mathrm{~A} \mathrm{MeV}$. We also plot in Fig. 3 some parametrizations taken from recent works and currently used in transport models [14,28-31]. They give rather different results in the Fermi energy domain, showing that $\sigma_{N N}^{i n-m e d i u m}$ is poorly constrained at present time. The parametrization of the $M S U$ group [31] is in excellent agreement -within the error bars- with our experimental findings.

\section{Conclusions}

We have evaluated nuclear stopping and transport features from measured isotropy ratio for protons in central collisions for a large body of symmetric systems studied with $I N D R A$ array. We have shown that we can get consistent results by scaling the appropriate stopping ratio by the characteristic size of the system. We have established a relation between the stopping ratio and the nucleon mean free path in nuclear matter and have found $\lambda_{N N}=9.5 \pm 2 \mathrm{fm}$ at $E_{i n c}=40 \mathrm{AMeV}$ and $\lambda_{N N}=4.5 \pm 1 \mathrm{fm}$ for $E_{\text {inc }}=100 \mathrm{~A} \mathrm{MeV}$, in agreement with theoretical predictions. We have also estimated in-medium effects for the nucleon-nucleon cross section. The best parametrization is the one provided by Danielewicz [31]. We conclude that in-medium effects give a significant reduction of the nucleon-nucleon cross section, namely $80 \%$ at $E_{\text {inc }}=35 \mathrm{~A} \mathrm{MeV}$ and $50 \%$ at $E_{\text {inc }}=100 \mathrm{~A} \mathrm{MeV}$. This strong energy dependence for the in-medium nucleon-nucleon cross section has to be properly taken into account in any transport model based on Boltzmann equation, where a 2-body collision term is considered.

\section{References}

[1] A. Ohnishi and J. Randrup, Phys. Rev. Lett. 75, 596 (1995).

[2] J. Aichelin, Phys. Rep. 202, 233 (1991).

[3] A. Bonasera et al., Phys. Rep. 243, 1 (1994). 
[4] P. Chomaz, M. Colonna, A. Guarnera and J. Randrup, Phys. Rev. Lett. 73, 3512 (1994).

[5] A. Ono, H. Horiuchi, T. Maruyama and A. Ohnishi, Phys. Rev. Lett. 68, 2898 (1992).

[6] T. Gaitanos et al., Phys. Lett. B 609, 241 (2005).

[7] S. Kumar, S. Kumar and R.K. Puri, Phys. Rev. C 81, 014601 (2010).

[8] C. Fuchs and H.H. Wolter, Eur. Phys. J. A 30, 5-21 (2006) and refs. therein.

[9] J.M. Lattimer and M. Prakash, Science 304, 536 (2004).

[10] G. Lehaut et al. (INDRA collaboration), Phys. Rev. Lett. 104, 232701 (2010).

[11] D. Durand, B. Tamain and E. Suraud, Nuclear Dynamics in the nucleonic regime, Institute Of Physics, New York (2001) and refs. therein.

[12] J. Cugnon, Ann. of Phys., Paris, Vol. 11 (1996).

[13] J. Lukasik et al. (INDRA and ALADIN collaboration), Phys. Lett. B 608, 223230 (2005).

[14] G. Q. Li and R. Machleidt, Phys. Rev. C 48, 1702 (1993).

[15] T. Frick, H. Muther, A. Rios, A. Polls and A. Ramos, Phys. Rev. C 71, 014313 (2005).

[16] V. Soma and P. Bozek, Phys. Rev. C 78, 054003 (2008).

[17] A. Rios and V. Soma, Phys. Rev. Lett. 108, 012501 (2012).

[18] G.D. Westfall et al., Phys. Rev. Lett. 71, 1986 (1993).

[19] A. Andronic et al., Eur. Phys. J. A 30, 31 (2006) and refs. therein.

[20] J. Pouthas et al., Nucl. Inst. and Meth. A 357, 418-442 (1995).

[21] G.Q. Zhang et al., Phys. Rev. C 84, 034612 (2011).

[22] S.K. Charagi and S.K. Gupta, Phys. Rev. C 41, 1610-1618 (1990).

[23] O. Lopez et al. (INDRA Collaboration), ArXiv:nucl-ex/1409.0735 (2014), submitted to PRC. 
[24] P.U. Renberg, D.F. Measday, M. Pepin, P. Schwaller, B. Favier, and C. Richard-Serre, Nucl. Phys. A 183, 81-104 (1972).

[25] A. Nadasen et al., Phys. Rev. C 23 1023-1044 (1981).

[26] K. Kikuchi and M. Kawai, Nuclear matter and Nuclear Collisions, North Holland, New York (1968)

[27] N. Metropolis et al., Phys. Rev. 110, 204-220 (1958).

[28] D. Klakow, G. Welke, and W. Bauer, Phys. Rev. C 48, 1982-1987 (1993).

[29] A. Schnell, G. Ropke, U. Lombardo, and H.J. Schulze, Phys. Rev. C 57, 806-810 (1998).

[30] C. Xiangzhou et al., Phys. Rev. C 58, 572-575 (1998).

[31] P. Danielewicz, Acta. Phys. Pol. B 33, 45 (2002). 